\title{
Choice, Infinity, and Negation: Both Set-Theory and Quantum-Information Viewpoints to Negation
}

\author{
Vasil Penchev, vasildinev@gmail.com \\ Bulgarian Academy of Sciences: Institute of Philosophy and Sociology: \\ Dept. of Logical Systems and Models
}

\begin{abstract}
The concepts of choice, negation, and infinity are considered jointly. The link is the quantity of information interpreted as the quantity of choices measured in units of elementary choice: a bit is an elementary choice between two equally probable alternatives. "Negation" supposes a choice between it and confirmation. Thus quantity of information can be also interpreted as quantity of negations. The disjunctive choice between confirmation and negation as to infinity can be chosen or not in turn: This corresponds to set-theory or intuitionist approach to the foundation of mathematics and to Peano or Heyting arithmetic. Quantum mechanics can be reformulated in terms of information introducing the concept and quantity of quantum information. A qubit can be equivalently interpreted as that generalization of "bit" where the choice is among an infinite set or series of alternatives. The complex Hilbert space can be represented as both series of qubits and value of quantum information. The complex Hilbert space is that generalization of Peano arithmetic where any natural number is substituted by a qubit. "Negation", "choice", and "infinity" can be inherently linked to each other both in the foundation of mathematics and quantum mechanics by the meditation of "information" and "quantum information".
\end{abstract}

Key words: choice, entanglement, infinity, information, negation, quantum correlations, quantum information,

Thesis:

1. The concept of negation supposes always a choice between negation and confirmation. "Choice", "negation", and "infinity" are inherently linked to each other in the ontological foundation of mathematics. The disjunctive choice between confirmation and negation as to infinity can be chosen or not in turn: This corresponds to set-theory or intuitionist approach to the foundation of mathematics and to Peano or Heyting arithmetic.

2. This can be demonstrated by a quantum and information approach to the foundation of mathematics. The theorems of the absence of hidden parameters in quantum mechanics (Neumann 1932; Kochen, Specker 1968) correspond to the intuitionist "third" as to infinity. The mapping of any coherent state before measurement into its statistical ensemble after measurement implies the well-ordering theorem equivalent to the axiom choice and thus the classical rather than intuitionist negation as to infinity. The reconciliation of both above as to negation in quantum mechanics calls the concept of information as classical as quantum in the foundation as the quantity of choices and thus of possible negations. 


\section{Prehistory:}

Intuitionism allows of the "third" along with confirmation and negation as to "spreads", which can continue unlimitedly. Thus Heyting arithmetic can be interpreted equivalently as Peano (1889) arithmetic, in which the "excluded third" is suspended as to infinite sets of natural numbers. Heyting inlike Peano arithmetic is consistent (Smorynski 1973; Visser 1982; Kanckos $2010)$ and can underlie mathematics. Gentzen's proof of completeness $(1936 ; 1938)$ by transfinite induction as well as even Hilbert's finitism $(1931 ; 1934)$ is reducible to Heyting arithmetic (Scarpellini 1972; Sommer 1995; Towsner 2005) as a two-dimensional Peano arithmetic or as Peano arithmetic complemented by choice. Actual infinity in set theory can be anyway consistently treated by means a bit stronger than a single Peano arithmetic: e.g. Peano arithmetic complemented by transfinite induction (Gentzen); two independent Peano arithmetics, i.e. a twodimensional Peano arithmetic (Heyting arithmetic in intuitionism); Peano arithmetic complemented by unlimited choice (Hilbert's finitism by " $\varepsilon$-symbol"). They seem to be equivalent and thus infinity linked to choice and negation provable by Peano arithmetic.

\section{The quantity of information:}

It can be introduced as the quantity of choices measured in units of elementary choice. That unit is a bit, i.e. the elementary choice between two equally probable alternatives. As the quantity of choices and in virtue of the above consideration, the concept of information turns out to be just what lacks in Peano arithmetic to be able to ground mathematics. In other words, Peano arithmetic complemented by the concept of information as a measure of choices or negations can generalize the ways for the foundation of mathematics.

\section{The quantity of quantum information:}

Quantum mechanics can be entirely reformulated in terms of information introducing the concept and quantity of quantum information. Its unit is a quantum bit (qubit) usually defined as the normed superposition of two orthogonal subspaces of the complex Hilbert space. A qubit can be equivalently interpreted as that generalization of "bit" where the choice is among an infinite set or series of alternatives. The complex Hilbert space in turn can be represented as a series of qubits, which is infinite in general, and any "point" in it (a "wave function" in quantum mechanics) is a value of quantum information. Furthermore, the complex Hilbert space is that generalization of Peano arithmetic where any natural number is substituted by a qubit. Thus the complex Hilbert space utilized initially by quantum mechanics is also a natural synthesis of Peano arithmetic and the concept of information therefore able to underlie mathematics in the approach above. It can be interpreted also as a set, any element of which is Peano arithmetic, e.g. as the set of all infinite subsets of Peano arithmetic.

"Choice", "negation", and "information":

These three closely linked notions are able to represent the fundamental mathematical concept of infinity avoiding the problem about "actual infinity". Furthermore, they have a natural interpretation in an experimental science such as quantum mechanics.

Information is the quantity of choices measured in units of elementary choice whether a bit or a qubit. A bit can be further decomposed as complex relation between identifying confirmation and negation and opposing them. Then a qubit is the case where confirmation and negation cannot be 
disjunctively separated from each other thus generating an infinite transition in-between, and their identification corresponds to a coherent state.

Quantum correlations, entanglement, and negation:

Any quantum entity by itself, i.e. before measurement cannot be disjunctively separated from all the rest in the universe. The phenomena of entanglement represent that inseparability. They are a corollary from the absence of hidden variables in quantum mechanics. If any quantity of two entangled quantum entities is measured, the correlations between their values can exceed the maximal possible limit of correlation between physical quantities in classical physics therefore violating Bell (1964) inequalities. If the quantum entity and its complement to the universe are interpreted as confirmation and negation, the phenomena of entanglement correspond to the third allowed by intuitionism.

Infinity and the quantum interpretation of set theory:

Quantum information allows of linking set theory to quantum mechanics by the meditation of Peano arithmetic and the concept of information. Thus the crucial but rather controversial notion of actual information turns out to be interpreted and treated by methods of an experimental science such as quantum mechanics. Roughly speaking, mankind is already able to plan experiments about infinity.

The main conclusion:

"Negation", "choice", and "infinity" can be inherently linked to each other both in the foundation of mathematics and quantum mechanics by the meditation of "information" and "quantum information". Therefore they represent the "entanglement" of both scientific areas.

The references include classical papers by:

- Bell, John (1964)

- Gentzen, Gerhard $(1936 ; 1938)$

- Hilbert, David (1931; 1934)

- Kochen, Simon and Ernst Specker (1968)

- Neumann, Johan von (1932)

- Peano, Giuseppe (1889)

\section{Furthermore:}

Kanckos, A. (2010) "Consistency of Heyting arithmetic in natural deduction," Mathematical Logic Quarterly 56 (6): 611-624.

Sommer, R. (1995) "Transfinite induction within Peano arithmetic," Annals of pure and applied logic 76 (3): 231-289.

Towsner, H. (2005) "Epsilon substitution for transfinite induction," Archive for Mathematical Logic 44 (4): 397-412.

Smorynski, C.A. (1973) "Applications of Kripke models," in Troelstra, A. S. (ed.) Metamathematical investigation of intuitionistic arithmetic and analysis, Berlin, Heidelberg, New York, Springer, pp. 324-391.

Scarpellini, B. (1972) "Induction and transfinite induction in intuitionistic systems," Annals of Mathematical Logic 4 (2): 173-227.

Visser, A. (1982) "On the completeness principle: A study of provability in Heyting's arithmetic and extensions," Annals of Mathematical Logic 22 (3): 263-295. 